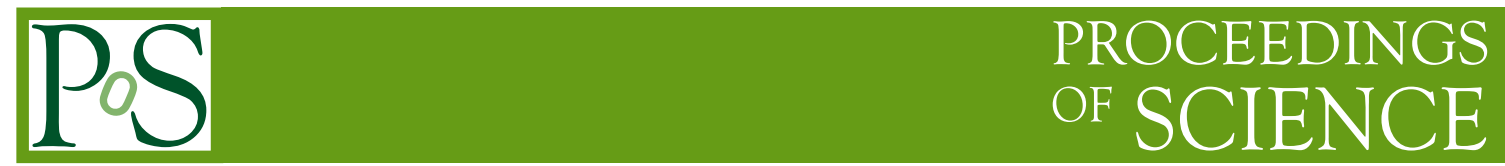

\title{
Search for the Standard Model Higgs boson at LHC
}

Fabio Cerutti - On behalf of the CMS and ATLAS collaborations.*

Laboratori Nazionali di Frascati - INFN

E-mail: fabio.cerutti@cern.ch

The Standard Model Higgs discovery potential of the ATLAS and CMS experiments is reviewed. Particular emphasis is given to four main discovery channels. The sensitivity to lower LHC center-of-mass energies is also discussed.

XXth Hadron Collider Physics Symposium

November 16 - 20, 2009

Evian, France

*Speaker. 


\section{Introduction}

The Standard Model Higgs is expected to be produced mainly via gluon-gluon fusion at the LHC p-p collider. For a center-of-mass energy of $14 \mathrm{TeV}$ the predicted cross-section is in the range of few tens of pb for masses between 114 and $200 \mathrm{GeV}$. The second production process in terms of cross-section is the so called Vector Boson Fusion (hereafter referred to as VBF) that is about one order of magnitude lower than the gluon-gluon fusion one. The main decay branching ratios for Higgs masses below $140 \mathrm{GeV}$ are in $b \bar{b}$ and $\tau^{+} \tau^{-}$final states. Above $140 \mathrm{GeV} \mathrm{WW}$ and $\mathrm{ZZ}$ final state are dominant, with a small deep of the $\mathrm{ZZ}$ branching ratio (BR) for masses around $170 \mathrm{GeV}$, due to the opening of the on-shell WW decay channel. A small decay branching ratio in $\gamma \gamma$ final state of the per mill order is predicted for masses in the range $120-140 \mathrm{GeV}$ and has a high experimental relevance as explained in Section 2. A direct 95\% CL lower limit on the SM Higgs mass of $114.4 \mathrm{GeV}$ has been determined at LEP [1]. An indirect 95\% CL upper limit on the same quantity of $157 \mathrm{GeV}$ can be derived from precision EW observables [2]. In the following four powerful discovery channels considered at the LHC are briefly described. The discovery is defined as the expected observation that has a probability of less than $2.9 \times 10^{-7}$ (i.e., the equivalent Gaussian integral probability above 5 Standard Deviations) to be compatible with an upward fluctuation of a background only experiment. In the low mass regime, below $130 \mathrm{GeV}$, the two main discovery channels are the $\gamma \gamma$ and the VBF $\tau \tau$ final states. For masses above $130 \mathrm{GeV}$ the $\mathrm{ZZ}^{*}$ and WW final states with both weak bosons decaying into leptonic final states are considered. Typical $\sigma \times B R$ of these four channels ranges from about $10 \mathrm{fb}$ for the $\mathrm{H} \rightarrow \mathrm{ZZ}^{*} \rightarrow 4 \ell$ up to about $1 \mathrm{pb}$ for the $\mathrm{H} \rightarrow \mathrm{WW} \rightarrow \ell v \ell v$ for masses above $130 \mathrm{GeV}$ (in this paper $\ell$ refers to electrons or muons). The other two processes qqH $\rightarrow \mathrm{qq} \tau^{+} \tau^{-} \rightarrow \mathrm{qq} \ell \nu v+x$ and $\mathrm{H} \rightarrow \gamma \gamma$ have expected $\sigma \times B R$ of the order of $100 \mathrm{fb}$ for masses close to $120 \mathrm{GeV}$.

The presence of a high transverse momentum $\left(\mathrm{p}_{T}\right)$ isolated photons or leptons in the final state ensures for all these channels an efficient trigger with a large rejection factor for pure QCD backgrounds, that are expected to be overwhelming at LHC. All the results reported in the following refers to an integrated luminosity between 10 and $30 \mathrm{fb}^{-1}$ at $14 \mathrm{TeV}$ and are based on recent ATLAS [3] and CMS [4, 5, 6, 7, 8] publications with the exception of the results of Section 6 where the potential at lower center-of-mass energies is explicitly discussed.

\section{Higgs to $\gamma \gamma$}

This channel is based on the search for a narrow mass peak in the two prompt-photons final state. Crucial ingredients for this search are the di-photon invariant mass, $\mathbf{M}_{\gamma \gamma}$, resolution and the jet versus prompt- $\gamma$ rejection. In fact backgrounds like di-jets and $\gamma$-jet with one photon from $\pi^{0}$ decay (the so called reducible background) have cross-sections that are up to six orders of magnitude higher than the irreducible background constituted of the two prompt-photons production. Both experiments expect a jet versus prompt- $\gamma$ rejection good enough to bring the reducible background well below the irreducible one. The mass reconstruction also requires the measurement of the photon directions. The best mass resolution is obtained when the direction of the photons is measured reconstructing the main Higgs production vertex with the Inner Detectors. In addition to the inclusive analysis some additional discriminant variables can be used to improve the signal over 
background ratio and the significance. To achieve this CMS uses in addition to the invariant mass other variables combined with multivariate techniques. The most powerful one is based on the photon transverse energies normalized to the photon pair invariant mass. An example of the expected di-photon invariant mass spectrum for the ATLAS inclusive analysis is shown in Figure 1 for the signal and the reducible and irreducible backgrounds. The ATLAS experiment has developed an

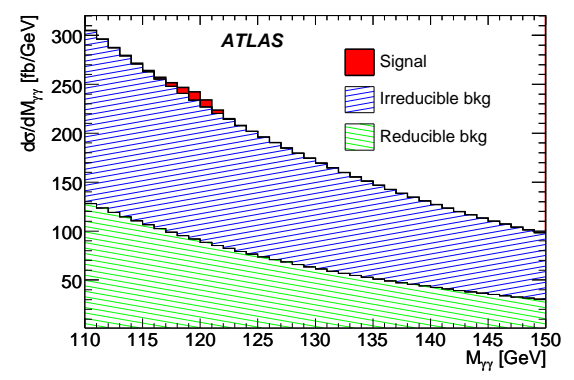

Figure 1: Expected $\gamma \gamma$ differential cross-section as a function of the $\gamma \gamma$ invariant mass. The signal is superimposed to the expected backgrounds.

analysis that exploits the presence in the final state of one or two high transverse-momentum jets. In fact the Higgs is expected to be produced in a good fraction of events in association with harder jet activity with respect to the background processes. In addition the data samples can be divided into categories that reflect the different mass resolution depending on the pseudorapidity of the photons and on the presence of conversions. All this information can be combined with multivariate techniques. It has to be stressed that it will be very difficult to get reliable background predictions, both in terms of normalization and shapes, from simulation. For this reason both experiments are planning to derive the background prediction from the invariant mass side-bands, outside the investigated signal region. The expected integrated luminosity needed to discover a Higgs with an invariant mass of $120 \mathrm{GeV}$ is of the order of $10 \mathrm{fb}^{-1}$ for the CMS experiment using optimized multivariate techniques. ATLAS expects to discover a $130 \mathrm{GeV}$ SM Higgs with an integrated luminosity of about $14 \mathrm{fb}^{-1}$. With $30 \mathrm{fb}^{-1}$ of integrated luminosity at $14 \mathrm{TeV}$ both experiments will be able to discover a SM Higgs for masses in the range between 120 and $140 \mathrm{GeV}$.

\section{Higgs to $\mathbf{Z Z}^{*} \rightarrow 4 \ell$}

The four leptons final states is the cleanest search channel for the SM Higgs at the LHC. This channel is characterized by a narrow peak of the four leptons invariant mass over a smooth background. The background is constituted of final states with four prompt leptons originating from $\mathrm{ZZ}^{*}$ production (irreducible background) and by final states in which at least two of the four leptons come from heavy-flavor decays or misidentified leptons like in the $t \bar{t} \rightarrow W b W \bar{b}$, the Zbb and the Z-jets processes. These reducible backgrounds can be rejected by requiring that the leptons are isolated, using calorimetric- and track-based isolation criteria, and prompt, requiring their compatibility with the main interaction vertex. With these experimental requirements the reducible background can be brought to a negligible level with respect to the irreducible one. At this level a 
simple inclusive analysis based on an invariant mass peak search over the continuous background is already very effective. Additional variables like the transverse momentum of the 4-leptons final state or angular variables that exploit the peculiar $J^{P C}$ quantum numbers of the SM Higgs could be used to improve the signal significance but both experiments have chosen not to use these variables in the discovery searches in order to keep the analysis more model independent. In this channel the

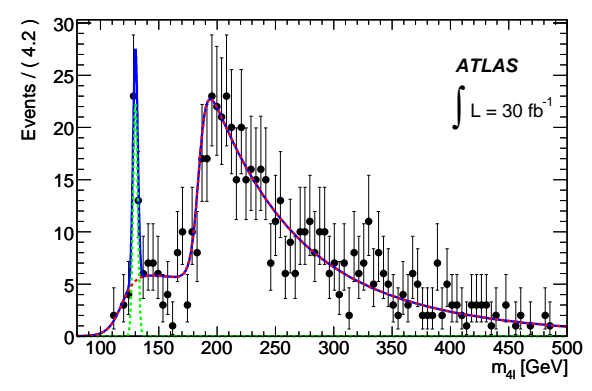

Figure 2: Invariant mass distribution of the 4-leptons final state for an ATLAS pseudo-experiment corresponding to an integrated luminosity of $30 \mathrm{fb}^{-1}$. A $130 \mathrm{GeV}$ Higgs signal is superimposed onto the SM backgrounds dominated by $\mathrm{ZZ}^{*}$ process. A fit to the signal plus background is superimposed.

background normalization can be derived by the mass side-bands so the background systematics is only relevant for the mass shape that is expected to be quite smooth with the exception of the region around the $\mathrm{ZZ}$ on-shell production (invariant masses around $180 \mathrm{GeV}$ ). An example of an ATLAS pseudo-experiment for a Higgs mass of $130 \mathrm{GeV}$ and an integrated luminosity of $30 \mathrm{fb}^{-1}$ is shown in Figure 2. With this channel a Higgs with a mass between 130 and $500 \mathrm{GeV}$ can be discovered with an integrated luminosity of about $30 \mathrm{fb}^{-1}$ with an exception for a Higgs of $170 \mathrm{GeV}$ where slightly more integrated luminosity is required.

\section{Higgs to WW with two leptons final state}

The $\mathrm{H} \rightarrow W W \rightarrow \ell v \ell v$ is the channel with the largest expected yield within the four considered in this paper. The presence of the two neutrinos in the final state doesn't allow the Higgs invariant mass reconstruction and therefore in this channel it is not possible to use the side-band methods to extract the background from the data. For this reason it is very important to develop methods based on data control samples to determine the background expectations and their systematic errors. This channel can be searched for in final states without additional hard hadronic activity (the so called O-jet final state) or in association with Vector Boson Fusion (VBF) production. The latter process is characterized by the presence of two energetic jets separated by a large pseudorapidity gap. Due to the fact that the Higgs is produced via the exchange of two weak bosons very little hadronic activity is expected in the pseudorapidity gap within the two tagging jets. The typical VBF tagging variables are therefore the requirement of two high-Pt jets with a large pseudorapidity gap, a large invariant mass of these two tagging jets and a jet-veto cut in the central detector region (pseudorapidity gap between the two tagging jets). The 0 -jet final state has a very high statistical power but is characterized by a relative small signal to background ratio. In this topology the dominant backgrounds are WW and $t \bar{t}$. The VBF final state has a more favorable signal over background ratio but 


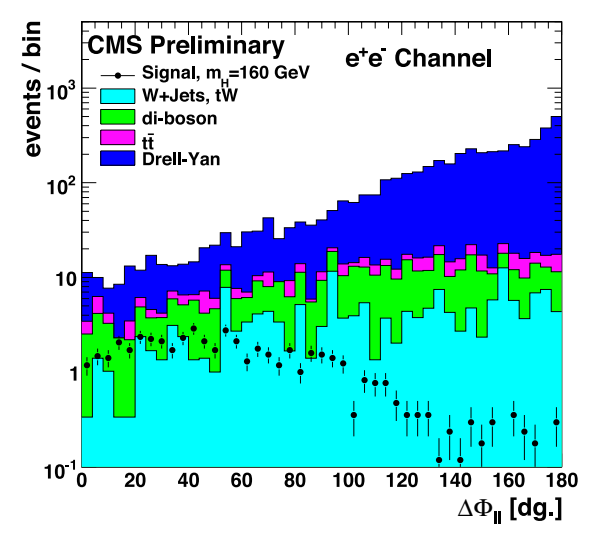

Figure 3: Expected $\Delta \phi_{\ell \ell}$ for the SM Higgs signal and main SM backgrounds in the $e^{+} e^{-}$final state from the CMS experiment. This figure refers to an integrated luminosity of $1 \mathrm{fb}^{-1}$ and is obtained after applying pre-selection and central jet veto cuts.

less statistical power due to the smallest production cross-section (about one order of magnitude lower than the gluon-gluon fusion process). Another very powerful discriminant variable is the angular separation between the two prompt leptons in the transverse plane $\Delta \phi_{\ell \ell}$. For the signal due to the scalar nature of the Higgs the two W's tend to have opposite spin orientations, which brings the two leptons to be more collinear in the transverse plane. The expected distribution for the $\Delta \phi_{\ell \ell}$ variable for the signal and for the main backgrounds from the CMS experiment is shown in Figure 3 for an integrated luminosity of $1 \mathrm{fb}^{-1}$ after applying pre-selection and central jet veto cuts (see [5]). This variable together with others like the two lepton invariant mass or the b-jet tag can be used to select signal-free control regions that are very similar to the final selected sample with the exception of one or two discriminant variables. In the control regions the background normalizations can be measured independently of the presence of a signal and then the simulation is used to extrapolate their yield in the signal region (just reversing the cut on the discriminant variable). In this way all the systematic uncertainty evaluation consists in estimating the possible error on the extrapolation factor based on simulation. It is therefore important to choose discriminating variables for which the shape is expected to be well reproduced by the simulation (both from the experimental and theoretical point of views). Both experiments have used control sample techniques to estimate expected error on the background normalization in the signal region. These uncertainties have been included into the significance evaluation. The results from CMS presented here are based on an inclusive analysis (dominated by gluon-gluon fusion production) while ATLAS has presented results for 0 -jet and VBF topologies separately but for the e $\mu$ final state only. The results from CMS shows that already with $1 \mathrm{fb}^{-1}$ of integrated luminosity a SM Higgs with a mass in the range between 155 and $175 \mathrm{GeV}$ can be discovered. The ATLAS discovery potential obtained by combining the 0 -jet and VBF final state shows a SM Higgs discovery in the range 140 to $190 \mathrm{GeV}$ for an integrated luminosity of $10 \mathrm{fb}^{-1}$. 


\section{Higgs to $\tau \tau$ with Vector Boson Fusion production}

The $\mathrm{qqH} \rightarrow \mathrm{qq} \tau^{+} \tau^{-} \rightarrow \mathrm{qq} \ell \nu v+x$ final state is important to complement the $\gamma \gamma$ final state in the low mass region. In this channel, thank to the high boost of the final state tau's (i.e., to the smallness of the tau-to-Higgs mass ratio) the Higgs mass can be reconstructed using the so called collinear approximation in spite of the presence of at least three neutrinos in the final state. The approximation consists of assuming that the direction of all the tau decay products is collinear. Under this assumption the two tau momenta can be reconstructed and the invariant mass resolution, dominated by the the missing transverse energy (Et-miss) resolution, is expected to be in the range between 10-15\%. The VBF signature, very similar to the one described in Section 4, helps in increasing the signal to background ratio. The main irreducible background for this channel is the $\mathrm{Z}$ inclusive production followed by $\mathrm{Z} \rightarrow \tau^{+} \tau^{-}$decay. The signal typically lay on the positive tail of the $\mathrm{Z} \rightarrow \tau^{+} \tau^{-}$invariant mass. For this reason is very important to have under control the mass shape of this background dominated by the Et-miss resolution. Both experiments have developed a technique that allows the extraction of this important quantity from the data. The technique requires the selection of $\mathrm{Z} \rightarrow \mu \mu$ decays using similar criteria as for the signal including VBF tag (but not the cut on Et-miss). Then the two real muons are substituted by two simulated taus with the same momenta. The shape of this emulated $\mathrm{Z} \rightarrow \tau \tau$ background has been shown to agree within better than $10 \%$ with the one from genuine $\mathrm{Z} \rightarrow \tau^{+} \tau^{-}$background. An example of this control distribution is shown in Figure 4 from CMS. With this channel a $115 \mathrm{GeV}$ Higgs is expected to

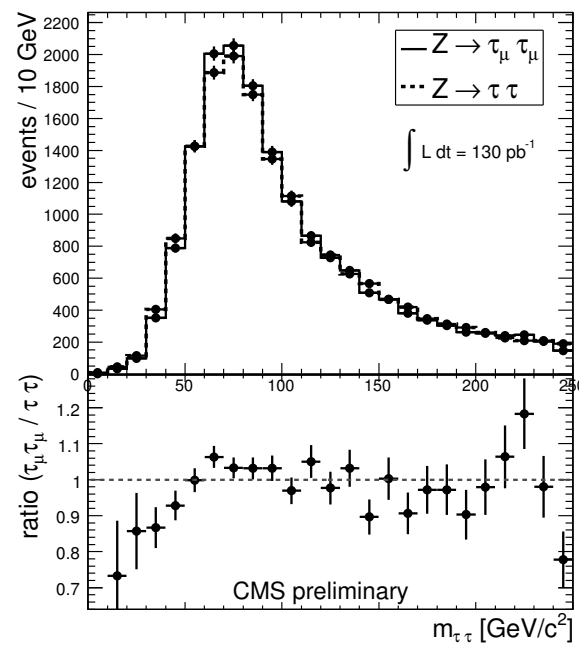

Figure 4: Reconstructed $\tau^{+} \tau^{-}$mass distribution for real (solid line) and emulated (dashed line) $\mathrm{Z} \rightarrow \tau^{+} \tau^{-}$ events from $\mu^{+} \mu^{-}$final state. In the lower plot the ratio between the two distributions is shown.

be discovered with an integrated luminosity of about $30 \mathrm{fb}^{-1}$ by a single experiment. In order to discover a SM Higgs with a mass up to $140 \mathrm{GeV}$ a slightly higher integrated luminosity, up to about $50 \mathrm{fb}^{-1}$, will be needed due to the reduced signal yield for higher masses. 


\section{Prospects at lower center-of-mass energies}

Some recent studies on the impact of a reduced LHC center-of-mass energy on the Higgs sensitivity have been presented by CMS for the WW and ZZ channels. The main effect is the reduction of signal and background cross-sections while the experimental acceptance is very slightly affected (at least for energy down to about $6 \mathrm{TeV}$ ). The impact on the signal and background cross-sections for center-of-mass energies of 10 and $6 \mathrm{TeV}$ is reported in Table 1.

\begin{tabular}{|c|c|c|}
\hline Process & $\frac{\sigma(10 \mathrm{TeV})}{\sigma(14 \mathrm{TeV})}$ & $\frac{\sigma(6 \mathrm{TeV})}{\sigma(14 \mathrm{TeV})}$ \\
\hline \hline$t \bar{t}$ & 0.450 & 0.113 \\
\hline $\mathrm{WW}$ & 0.650 & 0.320 \\
\hline $\mathrm{WZ}$ & 0.650 & 0.320 \\
\hline $\mathrm{ZZ}$ & 0.650 & 0.320 \\
\hline $\mathrm{gg} \rightarrow$ Higgs & 0.540 & 0.190 \\
\hline
\end{tabular}

Table 1: Expected cross section ratios for some relevant backgrounds and for the Higgs signal at 10 and 6 $\mathrm{TeV}$ LHC center-of-mass energies with respect to $14 \mathrm{TeV}$.

Considering the most pessimistic scenario (i.e., assuming all backgrounds scale like the WW one) the significance with respect to the one expected at $14 \mathrm{TeV}$ is reduced to about $70 \%$ for a center-of-mass energy of $10 \mathrm{TeV}$ and to about $30 \%$ for a center-of-mass energy of $6 \mathrm{TeV}$. This translates into a relative increase in luminosity needed to reach the same signal sensitivity of about a factor 2 at $10 \mathrm{TeV}$ and of about a factor 9 at $6 \mathrm{TeV}$. The CMS collaboration has shown that by combining the $\mathrm{ZZ}$ and WW channels with $1 \mathrm{fb}^{-1}$ of integrated luminosity collected at a center-ofmass energy of $10 \mathrm{TeV}$ a SM Higgs with a mass in the range between 150 and $190 \mathrm{GeV}$ can be excluded at $95 \% \mathrm{CL}$.

\section{Conclusions}

Four main discovery channels have been presented in this paper. Two of them, the $\gamma \gamma$ and the $\mathrm{ZZ} \rightarrow 4 \ell$, are very robust in terms of background determination thanks to the expected narrow mass peak over a smooth background in case of the presence of a signal. The other two requires the additional selection of control samples to estimate main backgrounds from the data. Both experiments developed possible strategies to cope with that. On the basis of the four channels described in this paper the two collaborations have shown that the SM Higgs can be excluded at $95 \% \mathrm{CL}$ for an integrated luminosity of about $2 \mathrm{fb}^{-1}$ collected at $14 \mathrm{TeV}$. An example of such an exclusion potential from the ATLAS experiment is shown in Figure 5. More details on this plot can be found in [3]. It has to be stressed that some of the channels used to achieve this result are based on very difficult experimental signatures like Et-miss and forward jet tag and therefore will require a very good understanding of the detectors that may take longer than the real time needed to collect such an integrated luminosity. Concerning discovery both experiment are expected to be able to cover the full mass range for an integrated luminosity of about $30 \mathrm{fb}^{-1}$ corresponding to about three years of data taking at an instantaneous luminosity of about $10^{33} \mathrm{~cm}^{-2} \mathrm{~s}^{-1}$. As an example the expected CMS significance for the SM Higgs as a function of the Higgs mass for an 


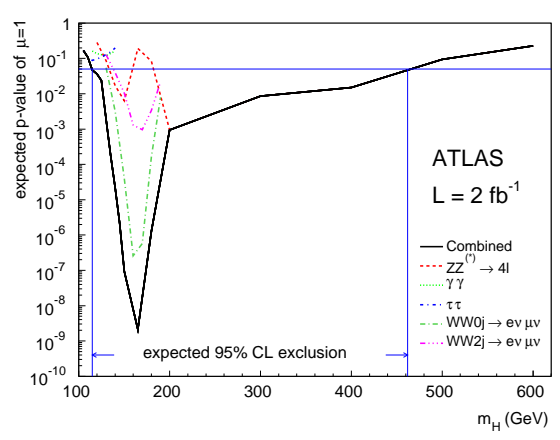

Figure 5: ATLAS expected p-value for a SM Higgs signal as a function of the Higgs mass. The solid line represents the combination of the channels listed on the picture. All masses with p-values below $5 \%$ are expected to be excluded at $95 \% \mathrm{CL}$.

integrated luminosity of $30 \mathrm{fb}^{-1}$ is shown in Figure 6. A more detailed explanation of this plot can be found in [4]. Several additional search channels not described in this paper are available at

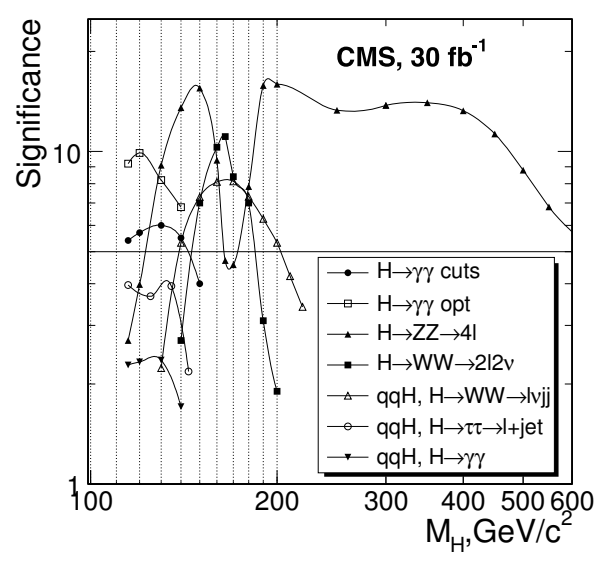

Figure 6: CMS expected SM Higgs signal significance as a function of the Higgs mass for an integrated luminosity of $30 \mathrm{fb}^{-1}$ collected at $14 \mathrm{TeV}$. Several search channels are shown together in this plot.

LHC and can be used to confirm a SM Higgs signal and measure its properties as described in [3] and [4].

\section{References}

[1] The ALEPH, DELPHI, L3 and OPAL collaborations, Search for the Standard Model Higgs boson at LEP, Physics Letters B 565, p.61-75, Jul 2003.

[2] The ALEPH, CDF, D0, DELPHI, L3, OPAL, SLD Collaborations. Precision Electroweak Measurements and Constraints on the Standard Model, CERN-PH-EP/2009-023.

[3] The ATLAS collaboration, Expected Performance of the ATLAS Experiment - Detector, Trigger and Physics, CERN-OPEN-2008-020. 
[4] The CMS collaboration, CMS Physics Technical Design Report, Volume II: Physics Performance, J. Phys. G: Nucl. Part. Phys. 34 995-1579.

[5] The CMS collaboration, Search Strategy for a Standard Model Higgs Boson Decaying to Two W Bosons in the Fully Leptonic Final State, CMS-PAS-HIG-08-006.- 2009.

[6] The CMS collaboration, Search for the Higgs boson in the ZZ* decay channel with the CMS experiment, CMS-PAS-HIG-08-003.

[7] The CMS collaboration, Towards the Search for the Standard Model Higgs boson produced in Vector Boson Fusion and decaying into a tau pair in CMS with 1 inverse femtobarn: tau identification studies, CMS-PAS-HIG-08-001.

[8] The CMS collaboration, Search for the Standard Model Higgs boson produced in Vector Boson Fusion and decaying into a tau pair in CMS with 1 inverse femtobarn, CMS-PAS-HIG-08-008. 